\title{
Predator Escape: An Ecologically Realistic Scenario for the Evolutionary Origins of Multicellularity
}

\author{
Jennifer T Pentz ${ }^{*}$, Tami Limberg ${ }^{2}$, Nicholas Beermann ${ }^{3}$ and William C Ratcliff ${ }^{*}$
}

\begin{abstract}
The origin of multicellularity was transformative for life on earth, allowing for the evolution of the large, complex organisms we see today. Despite its clear importance, the evolution of multicellularity remains a challenging topic to teach, especially at the high school level. Here we present a quick (one or two 50-min sessions) and easy laboratory in which students experimentally examine the hypothesis that small-mouthed predators can select for multicellularity. Students first observe rotifers and yeast (both unicellular and multicellular strains) separately under the microscope. Based on the observed natural history of the predator, they create a hypothesis for how predation will affect the survival of each type of yeast. They then test this hypothesis by feeding the yeast to the rotifers, quantifying the number of unicellular and multicellular yeast consumed, and analyze this result statistically. Pre- and post-lab assessment demonstrates the efficacy of our hands-on curricula in teaching fundamental concepts about multicellularity, as well as evolution in general.
\end{abstract}

Keywords: Multicellularity, Evolution, Biological complexity, Authentic curricula

\section{Background}

The evolution of complex life on Earth has occurred through key steps in which formerly autonomous organisms evolve to become integral parts of a larger, higherlevel organism. Smith and Szathmary (1995) termed these the major transitions in evolution, and they include the origin of eukaryotic cells from an endosymbiosis, the evolution of multicellular organisms, and even the evolution of 'superorganisms' composed of many multicellular entities. Multicellular organisms have independently evolved from unicellular ancestors at least 25 times in the history of life (Grosberg and Strathmann 2007), resulting in the origin of paradigmatic complex macroorganisms like plants and animals. Despite its fundamental importance in biology, early steps in this transition remain poorly understood. This is largely because

\footnotetext{
*Correspondence: jennifer.pentz@gatech.edu; william.ratcliff@biology. gatech.edu

${ }^{1}$ School of Biology, Georgia Institute of Technology, Atlanta, GA 30332, USA

Full list of author information is available at the end of the article
}

multicellularity arose a long time ago in Earth's extant lineages ( $>200$ million years ago) (Herron et al. 2009; Grosberg and Strathmann 2007), and early forms of multicellularity have been lost to extinction.

The transition to multicellularity involved a fundamental shift in the nature of biological organization (Buss 1987; Smith and Szathmary 1995). Single cells, formerly organisms in their own right, evolve to become parts in a new, larger individual (Buss 1987; Smith and Szathmary 1995). The first step in this transition was likely the evolution of simple multicellular clusters (Bonner 1998; Boraas et al. 1998; Kirk 2005; Pfeiffer and Bonhoeffer 2003). Once clusters evolve, the next step involves a shift in the level of selection, from single-cells to clusters (Damuth and Heisler 1988; Michod 2005). Here natural selection discriminates mainly between whole multicellular clusters, not between cells within them. If clusters possess the necessary ingredients for evolution via natural selection (that is, they possess heritable variation in multicellular traits and succeed or fail because of those traits), then between-cluster selection can result in the evolution of new cluster-level traits. This cluster-level 
adaptation can result in the evolution of increased multicellular complexity (Willensdorfer 2009; Bonner 2003).

Here we describe a novel laboratory experiment for high school and college students to study the very first step in the transition to multicellularity-the evolution of cellular clusters. Previous work has suggested that the advantages of increased size may have led to the evolution of multicellularity (Bonner 1965, 1998, 2000; King 2004). Proposed benefits include environmental protection (Gerhert and Kirschner 1997), cooperative metabolism (Dworkin 1972; Koschwanez et al. 2013), and immunity from predation by filter-feeding predators (Stanley 1973; Bonner 1988). Our research program uses the unicellular fungus baker's yeast (Saccharomyces cerevisiae) to study the evolution of multicellularity. Rather than focus on a selection mechanism underlying one of the above described hypotheses to select for increased size, we use a simple property of fluid dynamics: clusters of cells settle faster through liquid than single cells. This has proven to be a remarkably effective method of evolving multicellularity. Within just 60 days, clusters of cells (termed 'snowflake yeast') evolved and displaced their single-celled ancestors in all ten replicate populations studied (Ratcliff et al. 2012). We have previously described a student lab, modeled on the experiment of Ratcliff et al. (2012), in which students experimentally evolve multicellular yeast de novo (Ratcliff et al. 2014). Although this lab allows students to critically think about the evolution of multicellularity, it faces two significant challenges. First, experimental evolution requires substantial investments in both time (2 weeks of daily transfers) and resources (hundreds of tubes of sterile media), and few classes can work it into their schedules. Second, our selective agent (rapid settling though liquid media) was not chosen because we think that settling selection led to multicellularity in nature-we use it because it is a rapid and reliable method of selecting for larger size. Here we present a lab that circumvents both of these limitations, allowing students to examine how selection imposed by small-mouthed predators can favor the evolution of simple multicellularity.

\section{The Laboratory}

In this lab, students examine the ability for smallmouthed predators to select for simple multicellularity by assaying the survival of uni- and multicellular yeast that are fed to rotifers. In the first exercise, students observe the yeast (both uni- and multicellular) and rotifer separately, and formulate a prediction on if the rotifers will preferentially consume uni- or multicellular yeast. In the second exercise, students study the natural history of this interaction, examining how rotifers catch prey, and examining how both uni- and multicellular yeast fare in the face of predation. In the third exercise, students quantify the relative survival of uni- and multicellular yeast during predation, then analyze their results statistically.

\section{Materials}

The list of materials required for this lab can be found on page 2 of the teacher's guide (Additional file 1). The snowflake yeast kit can be obtained free of charge by emailing Prof. Will Ratcliff (will.ratcliff@biology.gatech.edu). Rotifers can be ordered from Carolina Biological Supply Company (Item \#133172, genus Philodina). We have included everything else required for this lab in the supplementary material: a student version of the lab manual (Additional file 2), two introductory PowerPoint presentations (Additional files 3, 4), videos of rotifer predation (Additional files 5, 6), pictures for quantifying rotifer predation (Additional file 7), and a pre-lab/post-lab test to assess student learning (Additional file 8 ). We intend to update this lab periodically, so please check our website (www.snowflakeyeastlab.com) for the latest curricular materials.

\section{The Prey and The Predator}

Unicellular and multicellular yeast (the prey) are supplied in the snowflake yeast lab kit, and have been fixed then stained with Congo red or methylene blue, respectively (Fig. 1a, b). These stains are toxic, so students should use gloves and protective eye gear while handling yeast. Rotifers (the predator) are small freshwater animals that prey on single-celled algae, bacteria, and ciliates (Nogrady et al. 1993). Commonly called 'wheeled animals', rotifers have a dense region of cilia on their head which creates a vortex, pulling food particles into their mouth (Fig. 1c, see Additional files 5, 6). Additionally, rotifers have a transparent body, allowing students to observe which type of yeast (based on its color) has been consumed (Fig. 1c).

\section{Exercise 1: Predicting Rotifer Predation}

To begin the lab, students start out by simply observing the rotifers and yeast separately. $100 \mu \mathrm{L}$ of Philodina rotifers are placed onto a depression slide and viewed on a compound microscope. Rotifers should be sucked up from the bottom of the container, as this is where they are the most concentrated. Students should pay special attention how the rotifers feed. Next, students view the yeast (included in the free kit) on the microscope by first diluting each stock culture 1:10 in water, then placing $5 \mu \mathrm{L}$ of each diluted culture on a standard slide. At this point, students create both a prediction and a hypothesis for how multicellular yeast should fare when fed to the rotifers relative to the unicellular strain. We believe that this is good practice, given how frequently predictions and hypotheses are conflated by students. If instructors 


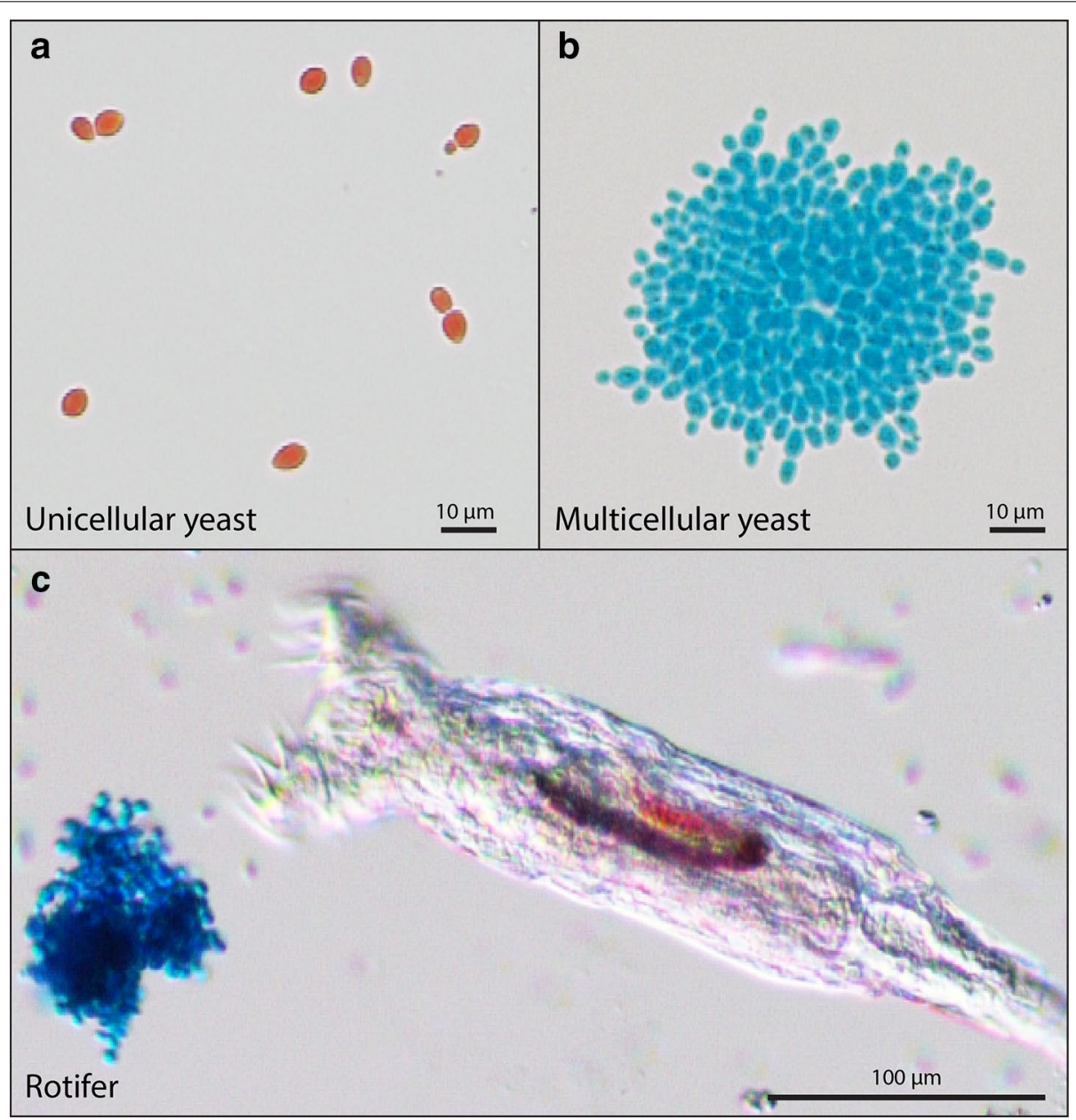

Fig. 1 Organisms used in this laboratory. a Unicellular yeast fixed and stained with Congo red. b Multicellular yeast fixed and stained with methylene blue. $\mathbf{a}, \mathbf{b}$ are provided in the snowflake yeast lab kit. c A rotifer, of the genus Philodina, when mixed with red unicellular yeast and blue multicellular yeast, will primarily eat red unicellular yeast (visible in the rotifers'stomach through its transparent body).

only have one 50 -min lab session to perform this lab, this entire observational section (Exercise 1) can be skipped to save time.

\section{Exercise 2: Observing Rotifer Predation}

Next, students feed the yeast to the rotifers to observe the effect of rotifer predation on the survival of uni- and multicellular strains. If the students have observed the rotifers and yeast separately (above), $5 \mu \mathrm{L}$ of each yeast culture can be added directly to the depression slide containing the rotifers, once again shaking the capped tubes vigorously to homogenize the yeast culture prior to use. Alternatively, students will add $100 \mu \mathrm{L}$ of Philodina rotifers and $5 \mu \mathrm{L}$ of each yeast culture together onto a depression slide. At this point, a coverslip can be added to the depression slide and the slide should be viewed immediately on a compound microscope.
Once the slide is on the microscope, students will observe the rotifers as they eat. Students should observe at least 10 feeding rotifers and examine which yeast is preyed upon more readily (Fig. 1c). Questions they can answer include: How do the rotifers eat? How long does it take for a rotifer to fill its stomach? What type of yeast (either uni- or multicellular) do the rotifers appear to prefer? Why might this be?

\section{Exercise 3: Quantifying Rotifer Predation}

In this experiment, students quantify the relative survival of uni- and multicellular yeast by counting the number of each type of yeast cell in five different rotifer stomachs. To begin, students transfer $10 \mu \mathrm{L}$ of the contents of the depression slide (in which rotifers have been feeding on the yeast) onto a standard microscope slide. Rotifers are flattened simply by placing a coverslip on top of the droplet. Next, students find five different rotifers and count the number 
of red cells and blue cells they see in their stomachs (Fig. 2). If students are having trouble with this step (many high school classrooms lack a sufficient number of high quality microscopes), students can use images of flattened rotifers provided in Additional file 7. To determine how well multicellular yeast survived predation, students calculate the fraction of total yeast consumed that were multicellular:

$$
\begin{aligned}
& \text { Proportion multicellular consumed } \\
& \quad=\frac{\# \text { of blue multicellular yeast }}{\text { \# of blue multicellular yeast }+ \text { \# red unicelluar yeast }}
\end{aligned}
$$

As your students will likely discover, rotifers strongly prefer to eat the unicellular yeast. Next, students examine the statistical robustness of this measurement by using a chi-square test. This simple statistical test compares the observed data with our expectations assuming that the rotifers do not have a preference for either kind of yeast. Because the rotifers were given equal numbers of unicellular and multicellular yeast to feed upon, the 'expected' number of either multi- or unicellular yeast consumed under the null hypothesis (no rotifer preference) is half of the total cell count. Students calculate the value of the chi-squared test statistic $\left(\chi^{2}\right)$ by summing the squared difference between the observed and expected number of uni- and multicellular yeast found in rotifer stomachs:

$$
\begin{aligned}
\chi^{2}= & \frac{\left(\# \text { Observed }_{\text {uni }}-\# \text { Expected }_{\text {uni }}\right)^{2}}{\# \text { Expected }_{\text {uni }}} \\
& +\frac{\left(\# \text { Observed }_{\text {multi }}-\# \text { Expected }_{\text {multi }}\right)^{2}}{\# \text { Expected }_{\text {multi }}}
\end{aligned}
$$

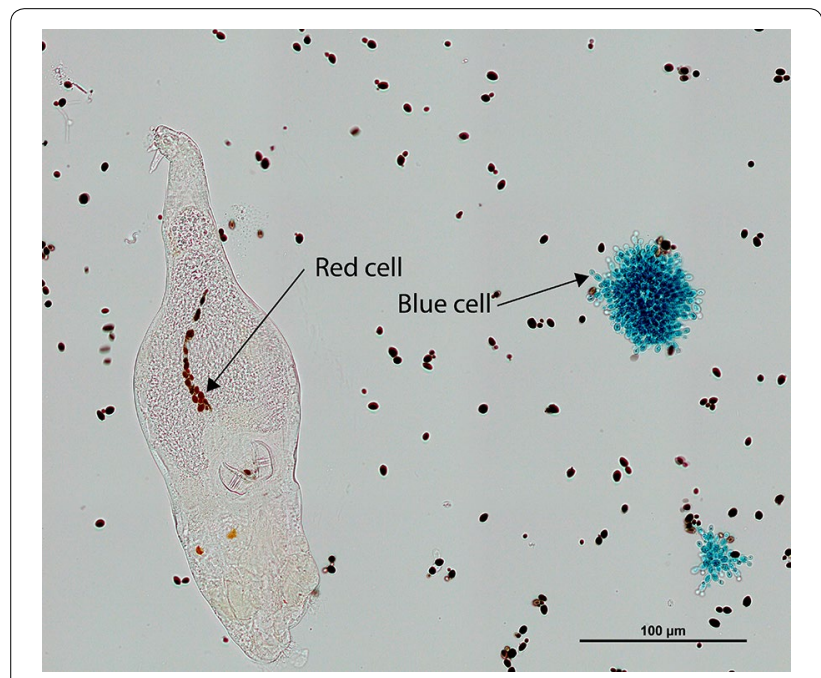

Fig. 2 Flattened rotifer. To quantify rotifer predation, students count the number of red and blue cells inside the rotifer's stomach (see arrows) after being flattened on a standard microscope slide. The transparent rotifer body allows for easy strain identification.
If the value obtained by the students is greater than 3.84 , then the result is significant at $p<0.05$, indicating that preference exhibited by the rotifers is statistically robust. While this equation might look a little daunting, we have found that most 9th grade biology students have little problem with it.

\section{Additional Exploration}

The above lab allows students to examine how predators can select for cellular clusters, the first step in the evolutionary transition to multicellularity. Observant students might point out that the lab has a chicken-and-egg problem: the rotifer predators are themselves a multicellular animal, so predation by a rotifer-like predator couldn't have led to the origin of the first multicellular organisms. We use rotifers in this experiment because they are large, slow moving, transparent (allowing observation of gut contents), and easy to handle. While they are not as easy to work with, unicellular predators (such as Paramecium aurelia) may be used instead of (or in addition to) rotifers. P. aurelia can be obtained from Carolina Biological Supply Company (Item \#131546). These predators exhibit many differences in their physiology and behavior (the most relevant being their nearly continuous and rapid movement, which makes microscopy difficult), but still strongly prefer to consume unicellular yeast. Like the rotifers, red and blue yeast cells can be counted in flattened $P$. aurelia, allowing students to statistically analyze feeding preference using eqs. 1 and 2. For a full description of how to use Paramecium in this lab, see Appendix 1 in Additional file 1.

\section{Assessment Results}

To determine the efficacy of our teaching labs, we administered pre- and post-lab assessments to students using this curricula in the introductory biology class Zoology at the University of Minnesota, and to high school students from six different institutions teaching at a range of levels (both AP and regular Biology). We do not have a sufficiently large sample size to separately analyze classes that performed just this lab, just our experimental evolution lab (Ratcliff et al. 2014), or both labs. Because both labs have similar teaching objectives and substantial curricular overlap, we combined these three lab options (e.g., experimental evolution lab, predator lab, or both) into a single analysis. In accordance with the Georgia Institute of Technology's IRB policies, we did not collect any data that would allow us to identify individual students. As a result, we were unable to use changes in individual student performance as our response variable. Instead, we compared overall performance on the pre-lab assessment to the post-lab assessment. Scores for individual questions were not normally 
distributed, so we compared the means of pre- and post-lab assessments using the nonparametric MannWhitney $U$ two-tailed test with a Bonferroni correction for multiple comparisons (Table 1). University students showed significant improvement in nearly all questions (all but question 7), but both smaller sample and effect sizes reduced our statistical power for high school students. As an additional comparative tool, we conducted 100,000 bootstrap simulations for each pre- and post-lab pair, comparing the fraction of runs in which the post-lab scores were higher than pre-lab scores (Table 1). Postlab assessment results were higher at least $95 \%$ of the time for all questions. These results suggest that student scores were indeed higher after the lab. The assessment covers a broad range of topics related to multicellularity, from the basic tenants of Darwinian evolution and how multicellularity evolves, to philosophical notions of biological individuality. The full list of questions can be found in Additional file 8. One question, which has been removed from our assessment, was not considered in this analysis (question not shown in Additional file 8). The question was poorly-chosen and did not address a learning objective of the labs.

Table 1 Statistical analysis of pre-lab and post-lab results

\begin{tabular}{|c|c|c|c|}
\hline $\begin{array}{l}\text { Question } \\
\text { number }\end{array}$ & $\begin{array}{l}\text { School } \\
\text { level }\end{array}$ & $\begin{array}{l}\text { Mann- } \\
\text { Whitney } \\
\text { U p value }\end{array}$ & $\begin{array}{l}\text { Fraction bootstrapped } \\
\text { runs in which } \\
\text { post-lab > pre-lab (\%) }\end{array}$ \\
\hline 1 & $\mathrm{HS}$ & $<0.0001^{*}$ & 100 \\
\hline 2 & $\mathrm{HS}$ & 0.0882 & 95.7 \\
\hline 3 & $\mathrm{HS}$ & $<0.0001^{*}$ & 97.1 \\
\hline 4 & $\mathrm{HS}$ & 0.0509 & 96.5 \\
\hline 5 & $\mathrm{HS}$ & 0.0463 & 96.9 \\
\hline 6 & $\mathrm{HS}$ & $0.0011^{*}$ & 97.3 \\
\hline 7 & $\mathrm{HS}$ & 0.0211 & 97.0 \\
\hline 8 & HS & 0.2357 & 95.7 \\
\hline 1 & Uni & $<0.0001^{*}$ & 99.9 \\
\hline 2 & Uni & $<0.0001^{*}$ & 99.9 \\
\hline 3 & Uni & $<0.0001^{*}$ & 99.9 \\
\hline 4 & Uni & $0.0027^{*}$ & 99.9 \\
\hline 5 & Uni & $0.0056^{*}$ & 99.9 \\
\hline 6 & Uni & $<0.0001^{*}$ & 99.9 \\
\hline 7 & Uni & 0.0465 & 97.9 \\
\hline 8 & Uni & $<0.0001^{*}$ & 98.2 \\
\hline
\end{tabular}

Scores for individual questions were not normally distributed, so we performed a non-parametric Mann-Whitney $\mathrm{U}$ test to compare the means of pre-lab and post-lab results (column 3 ).

* Denotes significance at the $\alpha=0.05$ level after Bonferroni correction for multiple-comparisons (threshold for significance is $p=0.05 / 8$ ). We ran 100,000 bootstrap simulations on each pre- and post-lab assessment and report the fraction of runs in which the post-lab score was greater than the pre-lab score (column 4). Sample sizes for HS pre-lab, HS post-lab, Uni pre-lab, and Uni postlab assessments were $242,119,423$, and 175 , respectively.
Overall, the labs appear to be quite effective at teaching higher-level concepts surrounding the transition to multicellularity. Post-lab scores were an average of $13.7 \%$ higher than pre-lab tests $(U=283632.5 ; p<0.0001$, MannWhitney $U$ test). As might be expected, undergraduate students displayed a better understanding of the material (Fig. 3). They averaged $65.5 \%$ correct on the pre-lab, in contrast to only $49.8 \%$ correct for high school students ( $U=60984.0 ; p<0.0001$; Mann-Whitney U test). In the post-lab assessment, undergraduates scored an average of $80.3 \%$ while high school students scored an average of $64.1 \%(U=12947.5 ; p<0.0001$; Mann-Whitney $\mathrm{U}$ test). High school students improved markedly on two

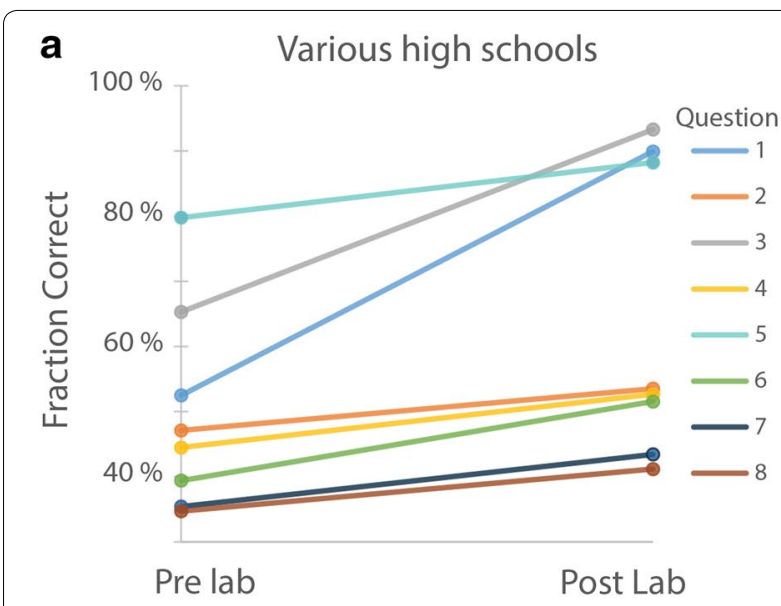

b University of Minnesota

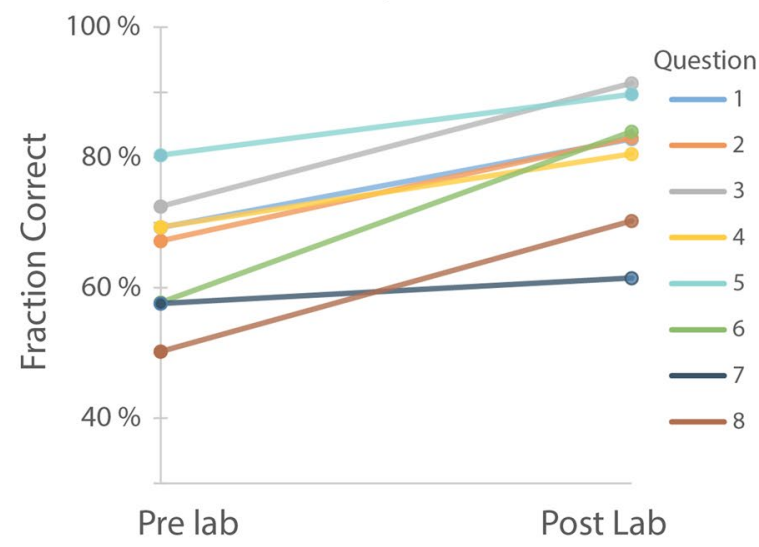

Fig. 3 Results of pre-lab and post-lab assessments for undergraduate and high school students. Overall, undergraduates scored higher on both pre and post-lab assessments. All students showed a substantial increase in their knowledge about the benefits of multicellularity (question 7). a High school students showed the most improvement in understanding the multiple origins of multicellularity (question 1) and cellular division of labor (question 2). b University of Minnesota undergraduates showed the largest increases in their understanding of multilevel selection (question 3) and biological individuality (question 8). 
questions: The first addressed the number of independent origins of multicellularity (question 1, Fig. 2a), while the second considered division of labor in multicellular organisms (question 2, Fig. 2a). University of Minnesota undergraduates improved dramatically on two different questions, the first concerning the steps required for multicellular organisms to evolve from single-celled ancestors (question 3; Fig. 2b), and the second on the topic of biological individuality (question 8; Fig. 2b). The latter may reflect the fact that the concepts taught about multilevel selection and the philosophy of individuality are the most advanced learning objectives in this lab, and probably more appropriate for university students.

The curricular materials associated with this lab include more than twenty questions to foster discussion and student thought. Because this lab involves predatorprey interactions, these discussions often touch on many other aspects of evolutionary biology and ecology, reinforcing the appropriateness of this lab in the introductory biology classroom. For example, one of the questions asks students to think about how the continued evolution of multicellular yeast will affect the evolution of the rotifers. Responding to this prompt, a 9th grade introductory biology student wrote:

"I think the unicellular yeast would start to evolve into multicellularity. This would happen as the multicellular yeast thrived while the unicellular yeast was suffering... Only the large rotifers with larger mouths (who could eat multicellular yeast) would be able to thrive. Natural selection would act on them and soon they would evolve to be larger and have larger mouths".

This student, a freshman in her first biology class, was able to figure out the concept of a co-evolutionary arms race from context of the lab and prompt alone.

\section{Discussion}

The major transitions in evolution, and macroevolution more generally, remain challenging topics to teach, especially in introductory biology classes. Indeed, the process through which radically new forms of life arise through Darwinian processes is hardly intuitive. This laboratory allows students to make the connection between macroevolutionary changes in morphology and the microevolutionary processes (e.g., mutation, selection, etc.) that underlie them. One hurdle that has long faced teachers trying to incorporate macroevolution into their curricula is the difficulty of exploring the subject with hands-on, organism-centric labs. Most labs exploring macroevolutionary change have relied on comparisons with living lineages, fossils, or computer simulations (Dubowsky and Hartman 1986; Rodrígues et al. Rodríguez et al. 2006; Soderberg and
Price 2003). While these are valuable teaching materials, inquiry based exercises that utilize living organisms can be more transparent, and offer a direct connection to the ecological theater in which evolutionary processes play out (Delpech 2009; Green et al. 2011; Olson and Loucks-Horsley 2000; Plunkett and Yampolsky 2010).

The lab described here incorporates hands-on exercises to teach key concepts about the evolution of multicellularity. In just two 50-min lab periods, students are able to examine how predation can lead to cluster formation, the first step in the transition to multicellularity. This is one of the central hypotheses for how multicellularity may have arisen in extant multicellular lineages (Bonner 1998). The lab incorporates observation and natural history, hypothesis formulation, collection of quantitative data, and statistical analysis, immersing students in the practice of science. In addition to teaching students about the ecological conditions that may have led to the origin of novel multicellular organisms, this lab teaches basic concepts about (1) Darwinian evolution, (2) the multilevel selection hypothesis for the evolutionary transition to multicellularity, (3) cellular division of labor, (4) benefits and costs of multicellularity, (5) and the history of multicellularity on Earth.

This lab addresses a number of Core Ideas and crosscutting concepts described in the Next Generation Science Standards (Schweingruber et al. 2012; see the NGSS page on our website for a more detailed discussion: http://www.snowflakeyeastlab.com/standards.htm). Core Idea LS1 "hinges on the unifying principle that cells are the basic unit of life." At its foundation, this lab is about the central role of cells in biology, as seen through the lens of the evolutionary transition to multicellularity. Core Idea LS2 examines the ecological interaction of organisms with their environment, including "how social interactions and group behavior play out within and between species". This lab addresses these issues directly, examining how predation, a between-species social interaction, can produce a selective pressure favoring group formation in yeast. While this lab does not allow students to examine evolution directly (for this see our experimental evolution lab module, Ratcliff et al. 2014), the lecture, readings, and discussion questions focus on evolutionary history and process. As a result, this lab teaches students about core ideas from LS3 (Heredity) and LS4 (Biological Evolution). Specifically, students will be taught about how microevolutionary processes can precipitate macroevolutionary change by applying Darwin's logic to cellular clusters (i.e., if clusters possess heritable genetic variation for multicellular traits, then selection acting on the properties of clusters can result in cluster-level adaptation). Finally, this lab emphasizes three cross-cutting concepts. First, students examine cause and effect through careful crafting and quantitative testing of a hypothesis for how 
multicellularity affects susceptibility to rotifer predation. Second, it teaches students to use model systems to explore big questions, and third, allows students to make connections between structure and function.

The bottom line: this lab is a fun, inexpensive, quick, and effective way to teach both high school and university students about macroevolution and the origin of biological complexity.

\section{Description of the Supplementary Files}

We have provided several resources to help teachers run this lab. Teacher and student guides contain a detailed overview of the lab with step-by-step instructions for students (Additional files 1,2). We provide two wellannotated introductory PowerPoint presentations for use introducing key background material on multicellularity and the lab activity, the first of which is detailed and may be more suited for university classes and AP biology (Additional file 3) while the second is a simpler version that may be better suited for some high school classes (Additional file 4). We provide two videos detailing rotifer predation (Additional files 5, 6). For schools with limited access to high quality microscopes, we include a set of images of flattened rotifers that can be used to quantitate rotifer predation preference in exercise 3 (Additional file 7). Finally, the pre-lab and post-lab assessment are included in Additional file 8.

\section{Additional Files}

Additional file 1: Laboratory teacher's guide. Teacher's guide for the lab that includes an overview of the lab, timeline for the lab, materials needed for the lab, detailed instructions for each lesson described in main text, additional exercises, and discussion questions for students.

Additional file 2: Laboratory student's guide. Student's guide for the lab that includes a detailed introduction to the concept of the transition to multicellularity, step-by-step instructions for each lesson described in the main text with tables for data entry, and discussion questions.

Additional file 3: Introductory PowerPoint (detailed). PowerPoint presentation for instructors to introduce the lab and the concepts of the transition from uni- to multicellularity. The presentation includes a brief introduction to multicellularity, snowflake yeast, and the predation lab.

Additional file 4: Introductory PowerPoint (simple). Simpler version of Additional File 3 if instructors feel that Additional file 3 is too detailed for their classroom. This may be more suitable for introductory high school classes.

Additional file 5: Rotifer predation video 1. Video of rotifer predation.

Additional file 6: Rotifer predation video 2. Video of rotifer predation.

Additional file 7: Images of flattened rotifers for quantifying predation. Multiple images of flattened rotifers for students to quantify rotifer predation if they are having trouble doing so through the microscope (see Lesson 2 in main text).

Additional file 8: Pre-lab and post-lab assessment. Pre-lab and post-lab assessment for instructors to give students to evaluate student's understanding of key concepts about the evolution of multicellularity before and after the lab.

\section{Authors' Contributions}

WCR, TL, and NB conceived of and designed the lab. JTP and WCR analyzed the data. JTP wrote the first draft of the paper (all authors, especially WCR, provided constructive feedback). All authors read and approved the final manuscript.

\section{Author details}

1 School of Biology, Georgia Institute of Technology, Atlanta, GA 30332, USA. ${ }^{2}$ Great River High School, St. Paul, MN 55108, USA. ${ }^{3}$ MacDowell Montessory High School, Milwaukee, WI 53213, USA.

\section{Acknowledgements}

This work was supported by the National Science Foundation grant no. NSF/ DEB1051115 AM3.

\section{Compliance with Ethical Guidelines}

\section{Competing Interests}

The authors declare that they have no competing interests.

Received: 22 March 2015 Accepted: 30 July 2015

Published online: 11 August 2015

\section{References}

Bonner, J. T. (1965). Size and cycle. Princeton: Princeton University Press.

Bonner, J. T. (1988). The evolution of complexity. Princeton: Princeton University Press.

Bonner, J. T. (1998). The origins of multicellularity. Integrative Biology: Issues, News, and Reviews, 1(1), 27-36.

Bonner, J. T. (2000). First signals: the evolution of multicellular development. Princeton: Princeton University Press.

Bonner, J. T. (2003). On the origin of differentiation. Journal of Biosciences, 28, 523-528.

Boraas, M. E., Seale, D. B., \& Boxhorn, J. E. (1998). Phagotrophy by a flagellate selects for colonial prey: a possible origin of multicellularity. Evolutionary Ecology, 12(2), 153-164.

Buss, L. W. (1987). The evolution of individuality. Princeton: Princeton University Press.

Damuth, J., \& Heisler, I. L. (1988). Alternative formulations of multilevel selection. Biology and Philosophy, 3, 407-430.

Delpech, R. (2009). Evolution in an afternoon: Rapid natural selection and adaptation of bacterial populations. Journal of Biological Education, 45, 54-59.

Dubowsky, N., \& Hartman, E. M, Jr. (1986). Simulated laboratory/field study of evolution. Journal of College Science Teaching, 15, 464-465.

Dworkin, M. (1972). The myxobacteria: new directions in studies of prokaryotic development. Critical Reviews in Microbiology, 2, 435-452.

Gerhart, J., \& Kirschner, M. (1997). Cells, embryos, and evolution: Toward a cellular and developmental understanding of phenotypic variation and evolutionary adaptability (p. 642). Oxford: Blackwell Sci.

Green, J. H., Koza, A., Moshynets, O., Pajor, R., Ritchie, M. R., \& Spiers, A. J. (2011). Evolution in a test tube: Rise of the wrinkly spreaders. Journal of Biological Education, 45, 54-59.

Grosberg, RK, \& Strathmann, RR (2007). The evolution of multicellularity: a minor major transition? Annual Review of Ecology, Evolution, and Systematics 621-654.

Herron, M. D., Hackett, J. D., Aylward, F. O., \& Michod, R. E. (2009). Triassic origin and early radiation of multicellular volvocine algae. Proceedings of the National Academy of Sciences USA, 106, 3254-3258.

King, N. (2004). The unicellular ancestry of animal development. Developmental Cell, 7, 313-325.

Kirk, D. L. (2005). A twelve-step program for evolving multicellularity and a division of labor. BioEssays, 27, 299-310.

Koschwanez, J. H., Foster, K. R., \& Murray, A. W. (2013). Improved use of a public good selects for the evolution of undifferentiated multicellularity. Elife, 2, e00367.

Michod, R. E. (2005). On the transfer of fitness from the cell to the multicellular organism. Biology and Philosophy, 20(5), 967-987. 
Nogrady, T., Wallace, R. L., \& Snell, T. W. (1993). Rotifera, Vol. 1: Biology, Ecology and Systematics. The Hague: SPB Academic Publishing.

Olson, S., \& Loucks-Horsley, S. (Eds.). (2000). Inquiry and the national Science education standards: A guide for teaching and learning. Washington, DC National Academy Press.

Pfeiffer, T., \& Bonhoeffer, S. (2003). An evolutionary scenario for the transition to undifferentiated multicellularity. Proceedings of the National Academy of Sciences USA, 100, 1095-1098.

Plunkett, A. D., \& Yampolsky, L. Y. (2010). When a fly has to fly to reproduce: selection against conditional recessive lethals in Drosophila. American Biology Teacher, 72, 12-15.

Ratcliff, W. C., Denison, R. F., Borrello, M., \& Travisano, M. (2012). Experimental evolution of multicellularity. Proceedings of the National Academy of Sciences, 109(5), 1595-1600.

Ratcliff, W. C., Raney, A., Westreich, S., \& Cotner, S. (2014). A Novel laboratory activity for teaching about the evolution of multicellularity. The American Biology Teacher, 76(2), 81-87.
Rodríguez, J., Ortiz, I., \& Dvorsky, E. (2006). Introducing evolution using online activities in a nonmajor biology course. Journal of College Science Teaching, 35, 31-35.

Schweingruber, H., Keller, T., \& Quinn, H. (Eds.). (2012). A framework for K-12 science education: Practices, crosscutting concepts, and core ideas. Washington, DC: National Academy Press.

Smith, J.M., \& Szathmary, E. (1995). The major transitions in evolution. Oxford University Press.

Soderberg, P., \& Price, F. (2003). An examination of problem-based teaching and learning in population genetics and evolution using EVOLVE, a computer simulation. International Journal of Science Education, 25, 35-55.

Stanley, S. M. (1973). An ecological theory for the sudden origin of multicellular life in the late Precambrian. Proceedings of the National Academy of Sciences USA, 70, 1486-1489.

Willensdorfer, M. (2009). On the evolution of differentiated multicellularity. Evolution, 63, 306-323.

\section{Submit your manuscript to a SpringerOpen ${ }^{\circ}$ journal and benefit from:}

- Convenient online submission

- Rigorous peer review

- Immediate publication on acceptance

- Open access: articles freely available online

- High visibility within the field

- Retaining the copyright to your article

Submit your next manuscript at $\gg$ springeropen.com 